\title{
ADAM10 promotes pituitary adenoma cell migration by regulating cleavage of CD44 and L1
}

\author{
Yuan Pan ${ }^{1,2}$, Chong Han ${ }^{3}$, Chunlin Wang ${ }^{1}$, Guohan Hu${ }^{1}$, Chun Luo ${ }^{1}$, Xiaoqiang Gan ${ }^{1}$, \\ Fenglin Zhang ${ }^{1}$, Yicheng $\mathrm{Lu}^{1}$ and Xuehua Ding ${ }^{1}$ \\ ${ }^{1}$ Department of Neurosurgery, Changzheng Hospital, Second Military Medical University, 415 Fengyang Road, Shanghai 200003, People's Republic of China \\ 2Department of Neurosurgery, the 401th Hospital of PLA, 22 Minjiang Road, Qingdao 266071, Shandong Province, People's Republic of China \\ ${ }^{3}$ Department of Endocrinology, Changzheng Hospital, Second Military Medical University, 415 Fengyang Road, Shanghai 200003, People's Republic of China \\ (Correspondence should be addressed to X Ding; Email: dingxuehuaczyy@163.com)
}

\begin{abstract}
ADAM10 is a metalloproteinase that regulates invasiveness in many tumors. Here, we found that $A D A M 10$ expression correlates with the invasiveness of pituitary adenomas and contributes to invasion by cleaving L1 and CD44. In highgrade pituitary adenoma patients, $A D A M 10$ expression levels were found to be elevated compared with low-grade pituitary adenomas. In a phorbol 12-myristate 13-acetate (PMA)-stimulated pituitary adenoma cell line, AtT-20 cells, we found that the cleavage of L1 was correspondingly enhanced with the increased interaction between Src and Shc. Increases in PMA-induced L1 cleavage and the phosphorylation of residue 418 of Src (418Src) were promoted by overexpression of ADAM10. Inversely, knockdown of Adam10 suppressed PMA-induced L1 cleavage and the phosphorylation of Src, which was blocked by the Src inhibitor PP2 and the MEK inhibitor PD98059. On the other hand, calcium flux activation in AtT-20 cells resulted in increased CD44 cleavage, with reduction of the interaction between calmodulin and ADAM10. The induction of enhanced CD44 cleavage by calcium flux activation was inhibited by knockdown of Adam10. In addition, Adam10 knockdown repressed AtT-20 cell migration, which was reversed by CD44EXT (CD44 ectodomain cleavage). Collectively, these data indicated that ADAM10 facilitated cell migration through modulation of CD44 and L1 cleavage.
\end{abstract}

Journal of Molecular Endocrinology (2012) 49, 21-33

\section{Introduction}

Human pituitary adenomas are tumors that occur in the pituitary gland and represent $\sim 10-15 \%$ of intracranial neoplasms. Pituitary adenomas are often considered to be benign tumors (Daniel et al. 2000). However, a proportion of these tumors do invade the surrounding tissues, including the sphenoid sinus, the cavernous sinus, and even the brain (Qian et al. 2007). Invasion of the cavernous sinus space occurs in $6-10 \%$ of all pituitary adenomas and has been shown to be the most common cause of incomplete tumor resection. Compared with noninvasive adenomas, tumors that invade the cavernous sinus show significantly higher proliferation rates (Knosp et al. 1993). In addition, a surgical cure becomes increasingly difficult with a higher grading score of the tumor. Cavernous sinus invasion implies an increased risk of intraoperative or postoperative cerebrospinal fluid leakage and of damage to the nerves in the cavernous sinus. However, the mechanisms underlying the invasiveness and malignancy of pituitary adenomas are poorly understood. Substantial evidence has indicated that no or only a few mutations of tumor suppressor genes or oncogenes are found in pituitary adenomas. Hence, we hypothesized that the abnormity of some adhesion molecules might be responsible for the aggressive behavior of pituitary adenomas.

The a-disintegrin-and-metalloprotease (ADAM) family is the major protein family that mediates ectodomain shedding with an activity similar to $\alpha$-secretase (Allinson et al. 2003). This family is a group of cell surface proteins that contains multiple domains. One of these domains is the metalloprotease domain, which can induce ectodomain shedding and extracellular matrix (ECM) cleavage. Another domain, the disintegrin domain, has been implicated in cell adhesion (Blobel 1997, White 2003). Ectodomain shedding has been described as the process by which membrane-attached cell surface proteins release their soluble ectodomains as a result of proteolysis at their extracellular sites proximal to the cell membrane. These released molecules can act as crucial regulators in many physiological and pathological events. The ADAM family is well known as the major sheddase and can shed a large variety of cell surface proteins, 
including growth factors, cytokines/cytokine receptors, and cell adhesion molecules (Mochizuki \& Okada 2007, Moss et al. 2008). Many of these substrates are involved in cancer. Consistent with this phenomenon, the upregulated expression of the members of the ADAM family has been observed in many cancers at both the mRNA level and the mature active protein level (Arribas et al. 2006, Santiago-Josefat et al. 2007). ADAMs have been regarded as a potential target for tumor therapy in breast cancer and small-cell lung cancer (Borrell-Pages et al. 2003, Zhou et al. 2006, Kenny \& Bissell 2007). ADAM10 belongs to the membraneanchored ADAM group and is ubiquitously expressed throughout most tissues (Seals \& Courtneidge 2003, Mochizuki \& Okada 2007, Moss et al. 2008, Parkin \& Harris 2009). The proteinase activity of ADAM10 contributes to Alzheimer's disease (Lammich et al. 1999, Allinson et al. 2003), inflammation, and cancer (Crawford et al. 2009, Pruessmeyer \& Ludwig 2009). Several reports have shown increased expression of ADAM1O in various types of cancer, including prostate cancer, colon carcinoma, and squamous cell carcinoma (Wu et al. 1997, Yoshimura et al. 2002, Fogel et al. 2003, McCulloch et al. 2004, Gavert et al. 2005, Ko et al. 2007). ADAM10 targets a series of adhesion molecules and the ECM, enhances the invasive properties of tumor cells, and facilitates tumor dissemination, which is the crucial step in cancer development and tumor progression (Millichip et al. 1998, Egeblad \& Werb 2002). However, whether ADAM10 is involved in pituitary adenomas remains unknown.

In this study, we analyzed a group of pituitary adenomas and found that the expression of ADAM1O correlated with tumor invasiveness and cleavage of CD44 and L1. CD44 and L1 cleavage significantly increased in highgrade tumors and was regulated by ADAM10 through calcium-dependent and -independent mechanisms. Calcium promoted ADAM10-mediated CD44 cleavage and reduced cell adhesion, at least partially through the depletion of the interaction of calmodulin (CaM) and ADAM10. Furthermore, phorbol 12-myristate 13-acetate (PMA) stimulation led to acceleration of the cleavage of L1, which is mediated by Src signaling. These data indicate that ADAM10 is involved in pituitary adenoma invasiveness by affecting cell adhesion and migration by cleaving CD44 and L1, suggesting that ADAM10 could be a potential target for clinical therapy.

\section{Materials and methods}

\section{Immunoblotting}

Cells were washed with chilled PBS and lysed with RIPA that contained a phosphatase inhibitor cocktail (Sigma) and protease inhibitors: $10 \mu \mathrm{g} / \mathrm{ml}$ phenylmethylsulphonyl fluoride, $10 \mu \mathrm{g} / \mathrm{ml}$ aprotinin, $10 \mu \mathrm{g} / \mathrm{ml}$ leupeptin, and $1 \mu \mathrm{g} / \mathrm{ml}$ pepstatin. After $30 \mathrm{~min}$ of chilled incubation, cell extracts were centrifuged at $15000 \mathrm{~g}$ for $15 \mathrm{~min}$ at $4{ }^{\circ} \mathrm{C}$. Then the supernatants were transferred, and the protein concentration was measured by the Bradford method. All samples were resuspended in an equal volume of $2 \times$ loading buffer. Equal amounts of protein were resolved using SDS-PAGE, transferred to nitrocellulose membranes, and incubated with the indicated antibodies. All immunoblots were visualized using a chemiluminescence detection kit (ECL; Santa Cruz Biotechnology, Santa Cruz, CA, USA).

The antibodies used in the experiments are as follows: anti- $\alpha$-tubulin (Sigma), anti-ADAM10 (Chemicon, Billerica, MA, USA), anti-L1 (Santa Cruz), anti-CaM (Upstate, Charlottesville, VA, USA), anti-CD44 (Ancell, Bayport, MN, USA), anti-p-418Src, anti-Src and antiShc (Cell Signaling Technology, Danvers, MA, USA), and HRP-conjugated goat anti-mouse and goat anti-rabbit secondary antibodies (Santa Cruz).

\section{Cell culture and treatment}

AtT-20 cells were maintained in F-12K medium (GIBCOBRL) with $2.5 \%$ heat-inactivated fetal bovine serum and $15 \%$ horse serum (GIBCO-BRL), streptomycin $(100 \mu \mathrm{g} / \mathrm{ml})$ and penicillin $(100 \mathrm{U} / \mathrm{ml})$ at $37^{\circ} \mathrm{C}$ in $95 \%$ air, and $5 \% \mathrm{CO}_{2}$. The cells were treated with dimethyl sulfoxide (control), PMA (100 ng/ml; Sigma), or ionomycin ( $5 \mu \mathrm{M}$; Calbiochem, San Diego, CA, USA). In some experiments, the cells were preincubated with the Src kinase inhibitor PP2 (preincubated for $1 \mathrm{~h}, 50 \mu \mathrm{M}$; Calbiochem) or the MAP kinase inhibitor PD98059 (preincubated for $1 \mathrm{~h}, 50 \mu \mathrm{M}$; Calbiochem). In other experiments, AtT-20 cells were treated with or without mechanical scraping or $100 \mu \mathrm{M}$ trifluoperizine (TFP, a CaM inhibitor, for $30 \mathrm{~min}$ ), after which cells were collected and subjected to further analysis.

\section{Human pituitary tissues and adenomas}

All samples were frozen and stored at $-80{ }^{\circ} \mathrm{C}$. Tumors were characterized based on their clinical, radiological, histological, and immunohistochemical features. Based on the secreting features, all tumors were classified as functional or nonfunctional. Tumor invasiveness was defined on the basis of preoperative radiological investigation using Knosp's classification (Knosp et al. 1993) and was confirmed during surgery. Grade 0-2 tumors were defined as noninvasive, while grade 3-4 tumors were considered to be invasive. Patient data regarding gender, age, tumor diameter, invasiveness, and secreting status are summarized in Table 1. Written informed consent was obtained from each patient. 
Table 1 The relationship between ADAM10, p-418Src, or p-ERK1/2 and clinicopathological features in pituitary adenomas $(n=90)$. Pearson's $\chi^{2}$ test

ADAM10

High Low

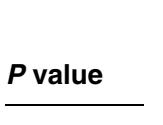

p-418Src

High Low

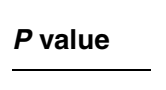

$\boldsymbol{P}$ value

$\begin{array}{ll}\text { Variable } & \\ \text { Age (years) } & \\ \leq 50 & 29 \\ >50 & 17 \\ \text { Gender } & \\ \text { Male } & 15 \\ \text { Female } & 31 \\ \text { Size (cm) } & \\ \leq 1 & 10 \\ 1-3 & 11 \\ \geq 3 & 25 \\ \text { Invasiveness } & \\ \text { Invasive } & 32 \\ \text { Noninvasive } & 14 \\ \text { Secreting } & \\ \quad \text { Functional } & 28 \\ \quad \text { Nonfunctional } & 18 \\ \text { Functional (PRL, } & 10 \\ 22 \text { cases) } & \\ \text { Functional (GH, } & 18 \\ \quad 38 \text { cases) } & \end{array}$

$\begin{array}{lllll}29 & 30 & 0.608 & 32 & 27 \\ 17 & 14 & & 17 & 1 \\ 15 & 11 & 0.426 & 11 & 1 \\ 31 & 33 & & 38 & 26 \\ 10 & 19 & 0.054 & 14 & 1 \\ 11 & 11 & & 12 & 10 \\ 25 & 14 & & 23 & 16 \\ 32 & 19 & 0.012 & 33 & 18 \\ 14 & 25 & & 16 & 23 \\ 28 & 32 & 0.233 & 34 & \\ 18 & 12 & & 15 & 1 \\ 10 & 12 & 0.886 & 14 & \\ 18 & 20 & & 20 & 18\end{array}$

$\begin{array}{rrrr}27 & 0.957 & 29 & 30 \\ 14 & & 16 & 15 \\ 15 & 0.141 & 12 & 1 \\ 26 & & 33 & 31 \\ 15 & 0.681 & 11 & 18 \\ 10 & & 12 & 10 \\ 16 & & 22 & 17 \\ 18 & 0.025 & 28 & 23 \\ 23 & & 17 & 22 \\ 26 & & & \\ 15 & 0.549 & 28 & 32 \\ 8 & & 17 & 13 \\ 18 & 0.407 & 7 & 15 \\ & & & \\ & & 21 & 17\end{array}$

30
5
14
31
18
10
17
23
22
32
13
15
17

$0 \cdot 824$

0.642

$0 \cdot 285$

0.288

0.371

0.079

\section{Immunohistochemistry}

Paraffin sections were obtained from the indicated patients. The sections were deparaffinized, and the epitopes were unmasked. After 30 min of blocking with serum, the sections were incubated with anti-ADAM10 antibody, anti-P-418Src antibody, and anti-ERK1/2 antibody (Cell Signaling) overnight at $4{ }^{\circ} \mathrm{C}$. The sections were then incubated with a biotinylated anti-rabbit antibody (Santa Cruz) for $30 \mathrm{~min}$ at $37^{\circ} \mathrm{C}$. Immunohistochemical staining was performed by the avidin-biotin complex method as the standard procedure.

\section{Immunocytochemistry}

AtT-20 cells grown on $35 \mathrm{~mm}$ culture dishes were fixed with $4 \%$ PFA for $15 \mathrm{~min}$ and incubated in $0.2 \%$ Triton X-100 in PBS for 5 min. After being washed with PBS, the cells were incubated in primary antibodies diluted in PBS containing $0 \cdot 2 \%$ BSA for $60 \mathrm{~min}$ at room temperature, washed three times in PBS, and incubated for $60 \mathrm{~min}$ at room temperature with the appropriate secondary antibodies linked to Cy5 diluted in PBS containing $0 \cdot 2 \%$ BSA. After being washed with PBS, the samples were mounted in $80 \%$ glycerol and visualized using a confocal microscope (model Fluoview; Olympus).

\section{Co-immunoprecipitations}

Homogenized tumor tissue or cultured cells were washed with ice-cold PBS and lysed for $30 \mathrm{~min}$ on ice using immunoprecipitation buffer (25 mM HEPES,
pH 7.4, $10 \%$ glycerol, $150 \mathrm{mM} \mathrm{NaCl}, 0 \cdot 1 \mathrm{mM}$ EGTA, $0.5 \mathrm{mM}$ dithiothreitol, and $0.5 \%$ Triton X-100) containing protease (Roche) and phosphatase inhibitor (Sigma) mixtures. The lysates were forced through a 26-gage needle four times and cleared by centrifugation at $16000 \mathrm{~g}$ for $10 \mathrm{~min}$ at $4^{\circ} \mathrm{C}$. Equal amounts of lysate were incubated with $2 \mu \mathrm{g}$ of the indicated antibodies together with $20 \mu \mathrm{l}$ protein A-Sepharose slurry overnight at $4{ }^{\circ} \mathrm{C}$ by continuous agitation. Immunocomplexes were washed four times in immunoprecipitation buffer, and precipitated proteins were eluted by boiling in Laemmli buffer. Proteins were separated using SDS-PAGE, transferred to polyvinylidene difluoride membranes, and incubated with antibodies. All immunoblots were visualized using a chemiluminescence detection kit (ECL; Santa Cruz Biotechnology). The antibodies used in the experiments are as follows: anti- $\alpha$-tubulin (Sigma), anti-ADAM10 (Chemicon), anti-L1 (Santa Cruz), antiCaM (Upstate), anti-CD44 (Ancell), and anti-Src and anti-Shc (Cell Signaling).

\section{Plasmid construction and transfection}

The sequence of ADAM10 was inserted between the KnpI and XhoI sites of the pENTR1A dual selection vector (Invitrogen) and then subcloned into pLenti6/V5-DEST (Invitrogen). The sequences of L1 and CD44 were inserted between the EcoRI and XhoI sites of the pENTR1A-GFP dual selection vector and then subcloned into pLenti6/V5-DEST. CD44EXT was 
generated by PCR from full-length CD44 cDNA and then subcloned into pLenti6/V5-DEST. The sequences (letters in bold indicate siRNA sequence after splicing) of shADAM10, 5'-CACCGAGATACATTAAAGATCGACGAA TCGATCTTTAATGTATCTCC-3' ${ }^{\prime}$ and 5'-AAAAGGAGATACATTAAAGATCGATTCGTCGATCTTTAATGTATCTC-3', were synthesized, annealed, and ligated into the vector pENTER/U6 (Invitrogen). Then, the pENTR/U6-ADAM10 0 shRNA plasmid was mixed with pLenti6/BLOCK-iT-DEST (Invitrogen) for an LR reaction to generate pLenti6/U6-ADAM10 ${ }^{\text {shRNA }}$.

Lentivirus was produced in 293FT cells by cotransfecting pLenti6/U6-ADAM10 $0^{\text {shRNA }}$ (pLenti6/ V5-DEST ${ }^{\text {ADAMIO }}$, pLenti6/V5-DEST ${ }^{\text {GFP-LI }}$, or pLenti6/ V5-DEST ${ }^{\text {GF-CD44 }}$ ) with the ViraPower Packaging Mix according to the manufacturer's instructions (Invitrogen). The media was changed $14 \mathrm{~h}$ later and the cells continued to incubate for 2 days. The media were collected and filtered through $0.45 \mathrm{~mm}$ sterile filters and directly added to the target cells.

Lentiviruses $(1 \mathrm{ml})$ were added to $35 \mathrm{~mm}$ dishes seeded with AtT-20 cells; after $12 \mathrm{~h}$, the media were replaced with another $1 \mathrm{ml}$ of fresh lentiviruses, and the cells were incubated for another $24 \mathrm{~h}$ for further experiments.

\section{HA coating and cell detachment assay}

Streptococcal hyaluronan ( $5 \mathrm{mg} / \mathrm{ml}$; Sigma-Aldrich) was added in a volume of $500 \mu \mathrm{l}$ to $35 \mathrm{~mm}$ dishes for $36 \mathrm{~h}$ at $4{ }^{\circ} \mathrm{C}$, then $500 \mu \mathrm{l}$ of $2 \%$ BSA was added, and incubated for $12 \mathrm{~h}$. The dishes were washed with F-12K medium, and AtT-20 cells were immediately seeded onto these dishes. Twenty-four hours later, shRNA transfection was performed and the cells were cultured for $\sim 3$ days. The cells were then treated with $5 \mu \mathrm{M}$ ionomycin in a serum-free medium for $2 \mathrm{~h}$. The suspended and adherent cells were collected, and the cell numbers were counted.

\section{Migration assay}

The filter inserts $(6.5 \mathrm{~mm}$-diameter polycarbonate filters of $8 \mu \mathrm{m}$ pore size, Corning) were coated with $1 \mathrm{mg} / \mathrm{ml}$ hyaluronan (Sigma-Aldrich) or $20 \mu \mathrm{g} / \mathrm{ml}$ fibronectin (Sigma-Aldrich) overnight at $4{ }^{\circ} \mathrm{C}$. The inserts were air-dried before the cells were added. Three days after infection, AtT-20 cells were digested and washed with FBS-free medium and then $2 \times 10^{4}$ cells were added to the top of the insert (in $100 \mu$ l of serumfree medium). F-12k medium (2.5\% FBS and $7 \cdot 5 \%$ horse serum) was added in a volume of $1 \mathrm{ml}$ to the lower chamber, the filter was placed into the lower chamber, and the cells were incubated at $37^{\circ} \mathrm{C}$. After $24 \mathrm{~h}$, inserts with the cells on the topside of the filter were removed and the cells on the lower surface were stained with Diff-Quik solution. The cells were washed twice, and the cell numbers in the lower compartments of the chambers were counted using a light microscope in five defined high-power fields.

\section{Statistical analysis}

Each experiment was repeated a minimum of three times, and the results were expressed as the mean \pm s.D. Statistical differences were determined using the $t$-test for the cell detachment and migration assays. The Pearson's $\chi^{2}$ test was used to assess the relationship between $A D A M 10, p-418 S r c$, and $p$-ERK1/2 expression and each clinicopathological factor and the correlation between p-418Src and p-ERK1/2 protein expression. Computations were performed using the SPSS 10.0 software (Aspire Software International, Leesburg, VA, USA). A $P$ value of $<0.05$ was considered significant.

\section{Results}

\section{Patient and tumor characteristics}

Of the 90 patients, 64 were females and 26 were males. The tumor size was $\leq 1 \mathrm{~cm}$ in 29 patients, $1-3 \mathrm{~cm}$ in 22 patients, and $\geq 3 \mathrm{~cm}$ in 39 patients. Fifty-one patients showed cavernous sinus invasion, while 39 patients had noninvasive tumors. Based on the secreting group classification, 60 patients were functional and 30 were nonfunctional adenomas (Table 1). Among 60 patients with functional adenomas, there are 38 cases of GH-secreting tumors and 22 cases of PRL-secreting tumors (Table 1).

\section{ADAM10 expression increased in high-grade pituitary adenomas and was associated with high levels of p-418Src and p-ERK1/2}

Substantial previous research has indicated that the expression level of $A D A M 10$ increases in many cancers. We evaluated $A D A M 10$ expression in pituitary adenomas by immunohistochemistry and found that $A D A M 1 O$ was expressed at high levels in high-grade pituitary adenomas compared with low-grade pituitary adenomas. Because p-418Src and p-ERK1/2 are involved in the cleavage of cell adhesion molecules, such as L1 (which might be driven by ADAM10), we also examined their expression levels in tumors from pituitary adenoma patients. High levels of expression of p-418Src and p-ERK1/2 were also observed in highgrade pituitary adenomas compared with low-grade pituitary adenomas (Fig. 1). Western blotting analysis of 

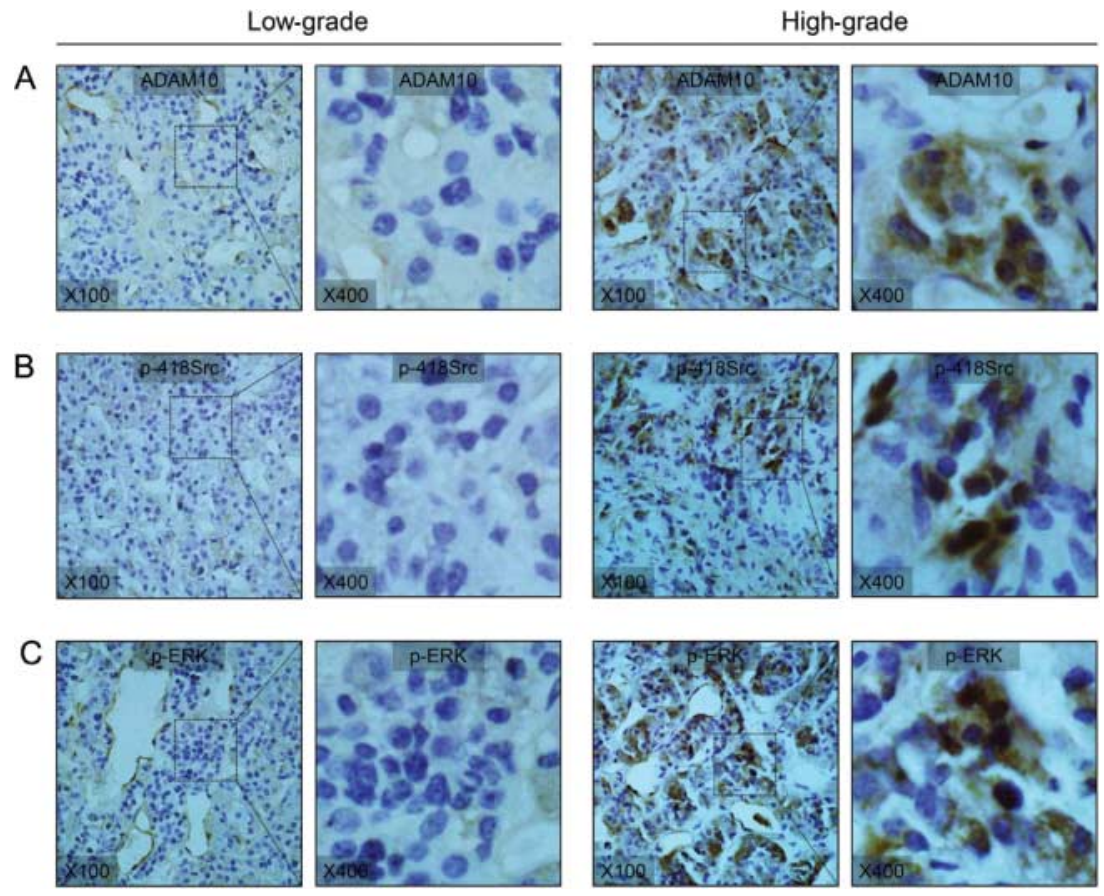

Figure 1 High levels of expression of $A D A M 10, p-418 S r c$, and $p-E R K 1 / 2$ are present in high-grade pituitary adenomas. ADAM10, p-418Src, and p-ERK1/2 are detected in low- and high-grade pituitary adenomas by immunohistochemistry.

the pituitary adenomas samples further confirmed the results (Fig. 2A and E).

To determine whether ADAM10 expression correlates with tumor invasion, we analyzed the relationship between ADAM10, p-418Src, p-ERK1/2, and clinicopathological features in 90 pituitary adenoma patients. We subdivided the adenomas into 'high'- or 'low'expressing ADAM10, full-length CD44, CD44EXT, p-418src, or p-ERK1/2 according to Kirkegaard's valuation standard (Kirkegaard et al. 2006), and tumors were subdivided into those with high (above the median) or low (below or equal to the median) expression. We found that ADAM10 expression correlates with the invasiveness of the tumor but has no relationship with the age or gender of the patient or the tumor size and secreting character of the tumor. The expression of $\mathrm{p}-418 \mathrm{Src}$, but not $\mathrm{p}$-ERK1/2, showed a similar relationship (Table 1 ). We also detected ADAM10, p-Scr, and p-ERK1/2 expression levels in the two subtypes of the functional pituitary adenomas respectively. The results revealed no obvious changes in the expression of three proteins between these two subtypes. We next analyzed the relationship between Src or ERK1/2 activation and the protein expression of ADAM10 in patient tumors. Our data indicated that the activation of Src and ERK1/2 correlated with ADAM1O expression (Table 2). These data suggest that ADAM10, together with p-418Src and p-ERK1/2, plays a role in high-grade pituitary adenomas.

\section{Cleavage of CD44 was increased in high-grade pituitary adenomas and was associated with increased $A D A M 10$ expression, which might be mediated by CaM and Src signaling}

To determine whether $A D A M 10$ expression levels were correlated with tumor invasiveness, we examined expression levels of CD44 in normal pituitary and in low- and high-grade pituitary adenomas, which is one of the important substrates of ADAM10 and plays the critical role in cell adhesion and migration (Gunthert et al. 1996, Schachner 1997, Brummendorf et al. 1998, Hortsch 2000, Murai et al. 2004). For the experiments, we chose 29 low-grade tumors with no cavernous sinus invasions and diameters below $1 \mathrm{~cm}$ and 39 high-grade tumors above $3 \mathrm{~cm}$ in diameter and with invasion in the cavernous sinus and surrounding the unilateral carotid artery.

Our data showed that Pro-ADAM10 expression was increased in high-grade pituitary adenomas, as was the cleavage of CD44 (Fig. 2A). However, no obvious changes were observed in normal pituitary and lowgrade pituitary adenomas (Fig. 2B). And the increased cleavage of CD44 correlated with high expression of Pro-ADAM10 (Table 3). These data suggested that ADAM10 exerts its function in high-grade pituitary adenomas, probably through CD44.

The calcium-dependent molecule CaM has been reported to interact with ADAM10 and suppress the 

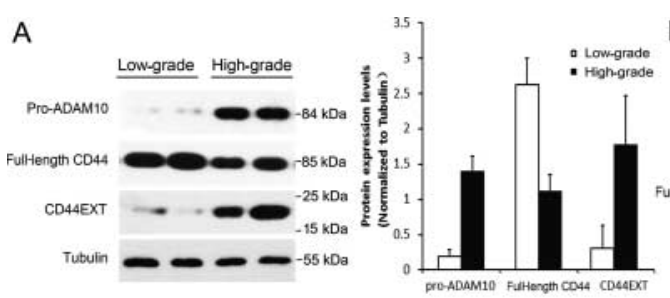

B
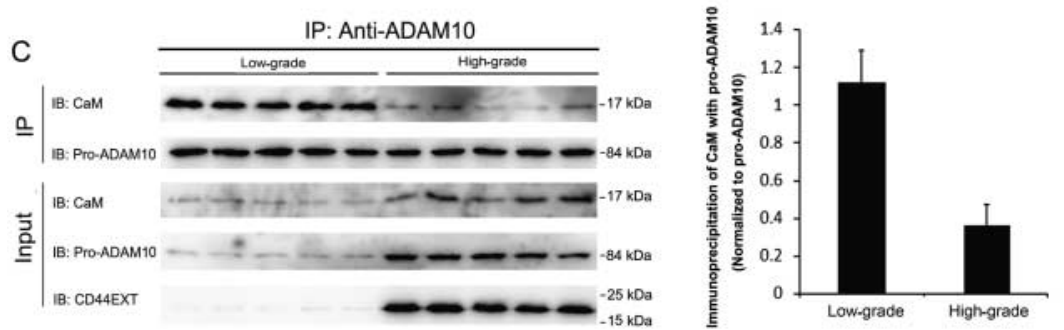

D
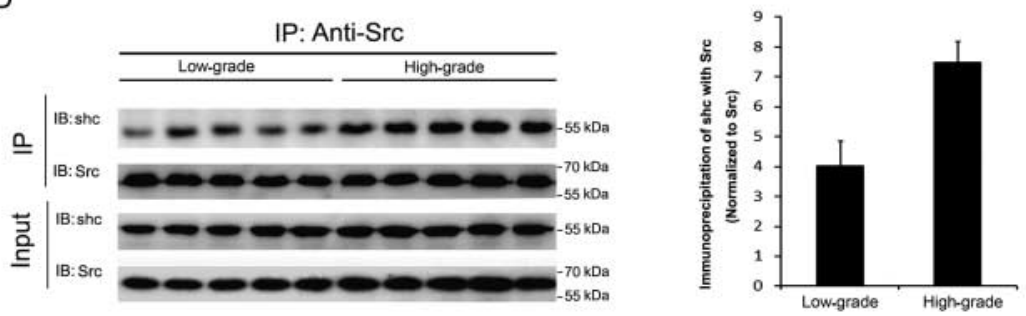

E
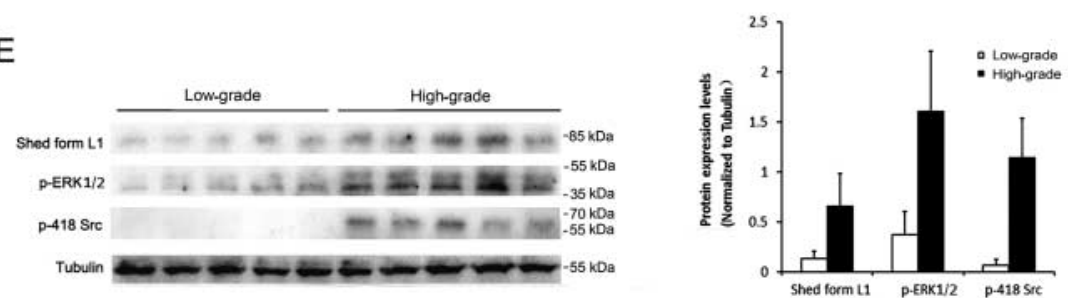

Figure 2 ADAM10/CaM interaction is reduced, and Src/Shc interaction is increased in high-grade pituitary adenomas expressing high level of $A D A M 10$, along with increased cleavage of CD44. (A) Typical examples of western blots for Pro-ADAM10, full-length CD44, and CD44EXT in pituitary adenomas. Tubulin is used as the internal control. Histogram of band densities comparing the amounts of the three proteins in the two different grade tumors, calculated from western blot experiments of 29 low-grade tumors and 39 high-grade tumors. (B) The protein expression levels of CD44EXT and full-length CD44 in normal pituitary tissues and pituitary adenomas. Tubulin is used as the internal control. (C) Pro-ADAM10 lost the interaction with CaM in high-grade pituitary adenomas, along with increased CD44EXT. Whole-tissue lysates were immunoprecipitated with anti-ADAM10 antibody and analyzed by immunoblotting with anti-CaM antibody and anti-ADAM10 antibody. Separate aliquots of the lysates were immunoblotted with the indicated antibodies. The histogram shows the relative amounts of CaM immunoprecipitated with Pro-ADAM10 in high-grade pituitary adenomas and low-grade pituitary adenomas. (D) Increased Shc interaction with Src in high-grade pituitary adenomas. Whole-tissue lysates were immunoprecipitated with anti-Src antibody and analyzed by immunoblotting with anti-Shc antibody and anti-Src antibody. Separate aliquots of the lysates were immunoblotted with the indicated antibodies. The histogram shows the relative amounts of Shc immunoprecipitated with Src in high-grade pituitary adenomas and low-grade pituitary adenomas. (E) The protein expression levels of shed form L1, $\mathrm{p}-\mathrm{ERK} 1 / 2$, and $\mathrm{p}-418 \mathrm{Src}$ in high-grade pituitary adenomas and low-grade pituitary adenomas. Tubulin is used as the internal control. Histogram of band densities comparing the amounts of the three proteins in the two different grades of tumors. 
ADAM10 cleavage function (Nagano et al. 2004). Therefore, we next examined the endogenous protein interaction between Pro-ADAM10 and CaM in low- and high-grade pituitary adenomas. A co-immunoprecipitation assay indicated that the interaction between Pro-ADAM10 and CaM is almost abolished in highgrade pituitary adenomas, which is associated with increased cleavage of CD44 (Fig. 2C). However, several groups have demonstrated that the interaction between Src and Shc can promote the activation of ERK1/2 (Barberis et al. 2000, Bulayeva \& Watson 2004, Kleiner et al. 2007), which has been reported to be a regulator of the ADAM10-mediated cleavage of L1 (Gutwein et al. 2000). Then, we next examined the interaction between Src and Shc in low- and high-grade pituitary adenomas using co-immunoprecipitation and found that the interaction between Src and Shc was increased in high-grade pituitary adenomas, which indirectly implied the increased L1 cleavage (Fig. 2D). The western blotting experiment result confirmed that cleavage of L1 was increased in high-grade pituitary adenomas (Fig. 2E). These data suggested that Pro-ADAM10 expression was increased in highgrade pituitary adenomas with increased cleavage of ADAM10 and CD44 and increased interaction between Src and Shc.

\section{ADAM10-induced L1 cleavage is mediated by SrC/ERK1/2 signaling}

L1 has important functions in cell adhesion and migration and can enhance invasiveness and metastasis (Schachner 1997, Brummendorf et al. 1998, Hortsch 2000). The overexpression of L1 has been observed in many tumor types (Ohnishi et al. 1998, Mechtersheimer et al. 2001, Thies et al. 2002). We also found that cleavage of L1 was increased in high-grade pituitary adenomas (Fig. 2E). To investigate the details of how ADAM10 regulates $\mathrm{L} 1$, we took advantage of the pituitary adenoma cell line AtT-20 to detect L1 cleavage. PMA, which is well known to induce L1 cleavage, was introduced into our assay. Our data showed that the

Table 2 The relationship between Src or ERK activation and the protein expression of ADAM10 in tumor specimens from 90 patients
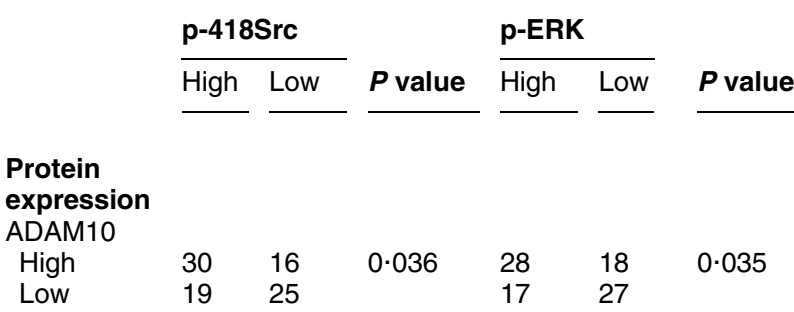

Table 3 The relationship between CD44 or CD44 cleavage and the protein expression of ADAM10 in 39 high-grade tumor specimens and 29 low-grade tumor specimens

\section{Full-length CD44}

$\underline{\text { High Low } P \text { value High Low }} \underline{P \text { value }}$

\section{Protein}

expression

ADAM10

High

Low

\begin{tabular}{lllll}
13 & 22 & 0.476 & 25 & 10 \\
19 & 14 & & 15 & 18 \\
\hline
\end{tabular}

0.602 phosphorylation of 418Src increases significantly in PMA-induced samples. This phenomenon is enhanced when ADAM10 is overexpressed. Moreover, when we pretreated the cells with the Src inhibitor PP2, the cleavage of L1 induced by PMA and the overexpression of ADAM10 were abolished (Fig. 3A). The opposite result was observed upon knockdown of the endogenous Adam10 expression by RNA interference (Fig. 3B). Similar results were also observed with the MEK inhibitor PD98059 (Fig. 3D). These data indicated that the cleavage of L1 induced by PMA is regulated by ADAM10. Src and ERK1/2 signaling participates in this process. Moreover, upon PMA stimulation, increased interaction between Src and Shc was observed (Fig. 3E). These results are consistent with those found in highgrade pituitary adenoma patients (Figs 1 and 2D). We also performed immunocytochemistry to detect the influence of ADAM10 on L1 cleavage. We found that L1 localizes on the cell membrane of unstimulated cells, and membrane L1 decreases with PMA treatment. However, upon Adam 10 knockdown, the membrane L1 did not decrease with PMA treatment, indicating that ADAM10 mediates L1 cleavage induced by PMA (Fig. 3F). Taken together, these data indicated that ADAM10 promotes L1 cleavage induced by PMA, and this process is at least partially mediated by the Src and ERK1/2 pathways.

\section{Calcium signals enhance ADAM10-induced CD44 cleavage}

CD44, another substrate of ADAM10, is a cell surface adhesion molecule that plays an important role in tumor invasion and metastasis (Gunthert et al. 1996, Murai et al. 2004) through the cleavage of its ectodomain. Calcium signals have been reported to activate the ADAM10-mediated cleavage of CD44 (Nagano et al. 2004). Our data also showed that ADAM10 activation is related to CaM, a calcium regulator protein. Here, we treated the cells by mechanical scraping, which can lead to the activation 


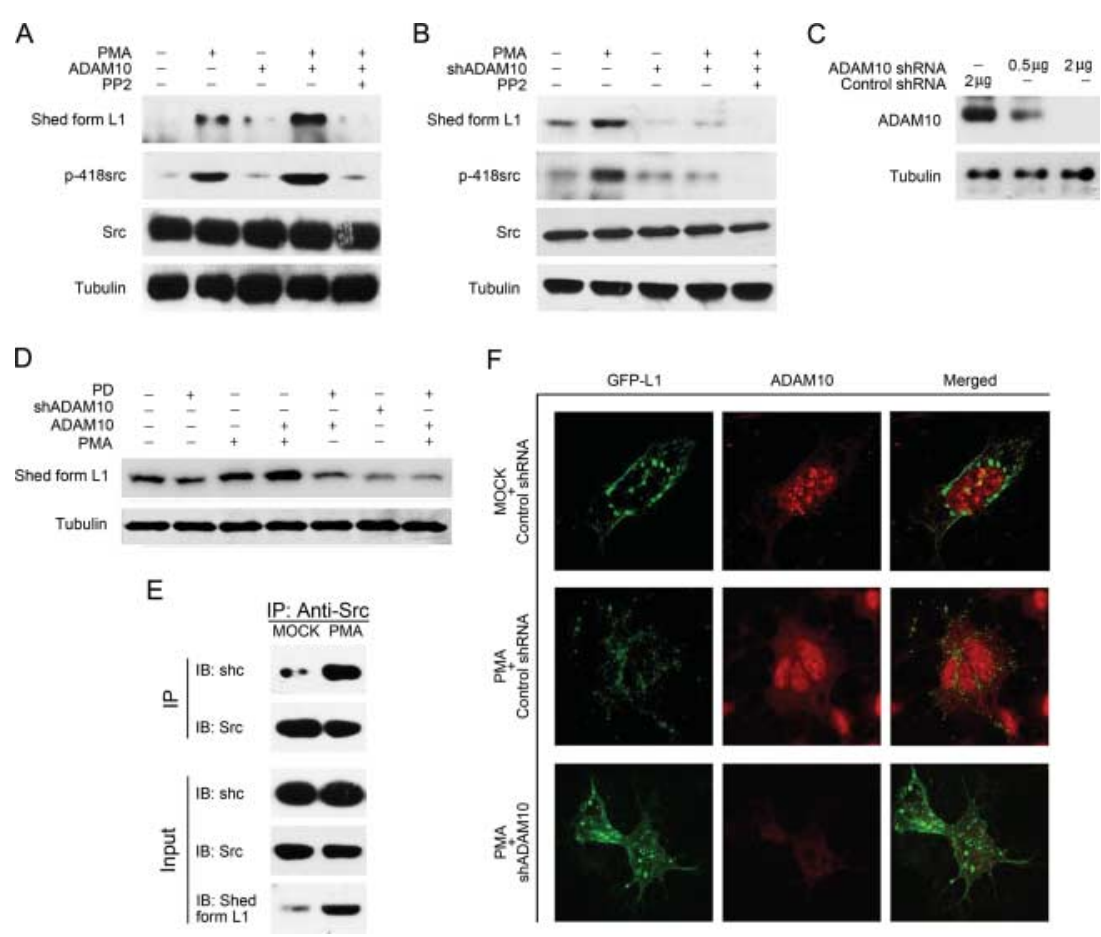

Figure 3 Src and ERK1/2 are involved in ADAM10-mediated L1 cleavage in PMA-treated AtT-20 cells. (A) PP2, a Src inhibitor, blocks the cleavage of L1 induced by PMA in ADAM10 overexpressing AtT-20 cells. AtT-20 cells were transfected with ADAM10 and treated as indicated earlier. Protein levels in the whole-cell extract were analyzed by western blotting. (B) The absence of ADAM10 blocks the cleavage of L1 induced by PMA in AtT-20 cells. AtT-20 cells were transfected with shADAM10 and treated as indicated earlier. (C) shADAM10 effectively reduces the expression of ADAM10. After transfection with shADAM10, the total cell lysate of AtT-20 cells was analyzed. (D) PD98059, an MEK inhibitor, further reduced the cleavage of $L 1$ induced by PMA in shADAM10-treated AtT-20 cells. AtT-20 cells were transfected with ADAM10 expression plasmid or shADAM10 and treated as indicated earlier. (E) Interaction between Shc and Src was increased in PMAtreated AtT-20 cells. Whole-tissue lysates were immunoprecipitated with anti-Src antibody and analyzed by immunoblotting with the indicated antibodies. $(F)$ Subcellular location of $L 1$ and ADAM10 in AtT-20 cells after PMA treatment. After transfection with the indicated plasmids and PMA treatment, the cells were observed by confocal microscopy.

of the cellular calcium signal (Nagano et al. 2004). After treatment, CD44 cleavage increased, while the expression of full-length CD44 decreased. This phenomenon could be blocked by the knockdown of Adam10 expression (Fig. 4A). Similar results were also observed with ionomycin treatment (Fig. 4B). Immunocytochemistry was also performed to detect the influence of ADAM10 on CD44 cleavage. With ionomycin treatment, less CD44 localized to the cell membrane compared with normal cells. Upon Adam10 knockdown, the membrane CD44 showed no difference compared with normal cells (Fig. 4D). These results suggested that ADAM10 regulates CD44 cleavage in calcium flux-stimulated pituitary adenoma cells. We then pretreated the cells with EGTA to chelate the calcium before ionomycin was added. We found that ADAM10 activation was blocked by EGTA (Fig. 4C), which suggests that calcium flux regulates ADAM10 activation. To further investigate how calcium flux regulates ADAM10 activation, we examined the interaction between Pro-ADAM10 and CaM. We observed that the interaction between Pro-ADAM10 and CaM decreases in high-grade pituitary adenomas, which is associated with increased CD44 cleavage (Fig. 2C). In the pituitary adenoma cell line treated by mechanical scraping or treated with the CaM inhibitor TFP, the interaction between $\mathrm{CaM}$ and ADAM10 was decreased significantly (Fig. 5A). CaM has been reported to suppress ADAM10 activation by protein interactions. Our results suggested that extracellular calcium flux decreases the interaction between Pro-ADAM10 and CaM. Taken together, our data indicated that ADAM10-mediated cleavage of CD44 depends on the cellular calcium signal and is at least partially mediated by the release of the CaM-mediated suppression of ADAM10. 


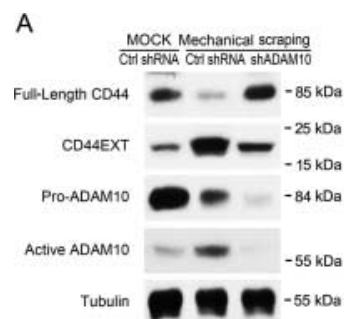

B

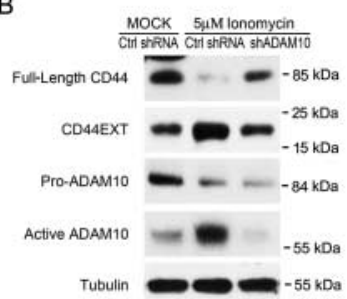

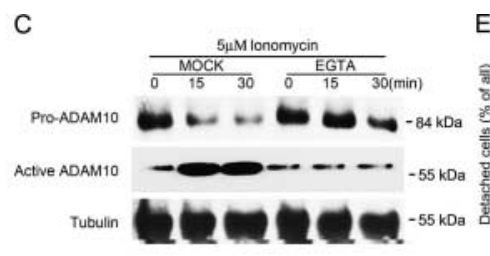
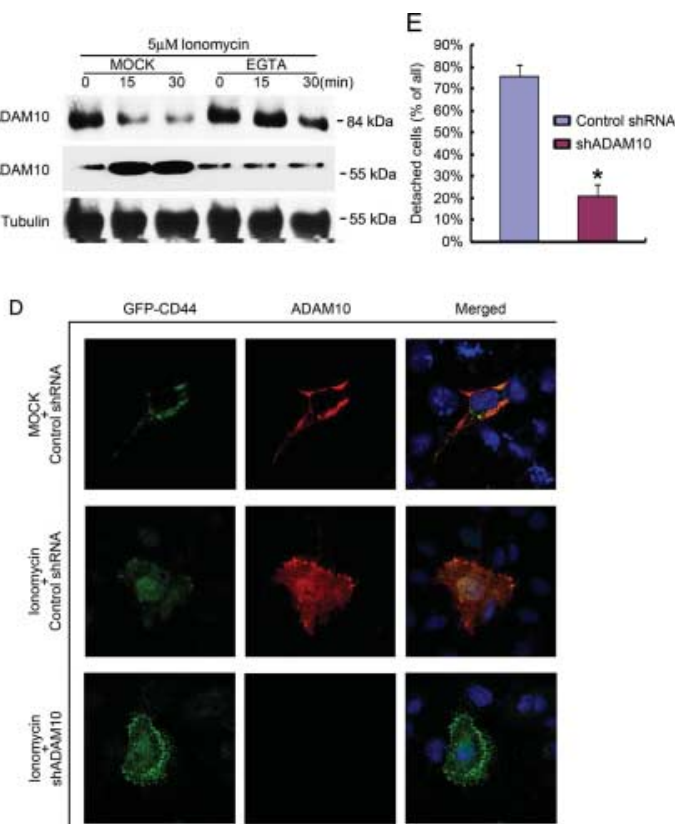

ADAM10
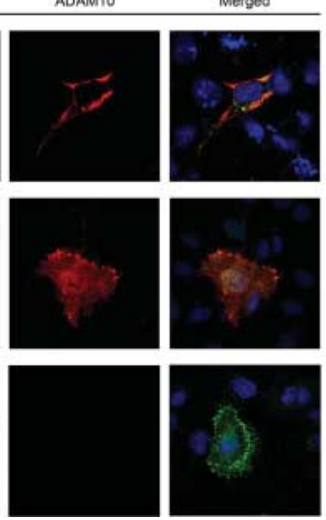

Figure 4 Calcium upregulates the active form of ADAM10, which increases the number of detached cells and causes CD44 cleavage. (A) The absence of ADAM10 blocks the cleavage of CD44 induced by mechanical scraping in AtT-20 cells. AtT-20 cells were transfected with the indicated shRNA and treated as indicated earlier. Protein levels in whole-cell extract were analyzed by western blotting. (B) The absence of ADAM10 blocks the cleavage of CD44 induced by ionomycin in AtT-20 cells. AtT-20 cells transfected with the indicated plasmids were treated with or without $5 \mu \mathrm{M}$ ionomycin for $1 \mathrm{~h}$. Protein levels in whole-cell extract were analyzed by western blotting. (C) EGTA prevents Pro-ADAM10 from becoming active ADAM10 in ionomycin-treated AtT-20 cells. AtT-20 cells were treated with or without $5 \mu \mathrm{M}$ ionomycin for $1 \mathrm{~h}$ in the absence or presence of $10 \mathrm{mM}$ EGTA. Protein levels in whole-cell extract were analyzed by western blotting. (D) Subcellular localization of CD44 and ADAM10 in AtT-20 cells after ionomycin treatment. AtT-20 cells transfected with the indicated plasmids were treated with or without $5 \mu \mathrm{M}$ ionomycin for $1 \mathrm{~h}$. The cells were then examined by confocal microscopy. (E) The absence of ADAM10 reduces the detachment of AtT-20 cells. ${ }^{*} P<0.05$.

\section{ADAM10 plays a role in regulating cell adhesion and migration, which is mediated by CD44}

We also examined whether ADAM10 is involved in the modulation of cell adhesion and migration. We used shRNA to knockdown Adam10 expression in pituitary adenoma cells and then compared the percentage of detached cells. The results indicated that cell adhesion increases significantly when ADAM1O expression is impaired (Fig. 4E). Next, we wanted to investigate whether the ADAM10-mediated regulation of cell adhesion and migration is dependent on cleavage of CD44. As CD44 can interact with hyaluronic acid, but not with fibronectin, we examined the cell migration ability under both hyaluronic acid- and fibronectincoated conditions upon Adam10 knockdown. We found that knockdown of Adam10 greatly repressed cell migration with hyaluronan but had no effect on cell migration with fibronectin (Fig. $5 \mathrm{~B}$ and $\mathrm{C}$ ). Importantly, overexpression of CD44EXT reversed the effect of Adam10 knockdown on cell migration. These results suggested that CD44 mediates the function of ADAM10 in the regulation of pituitary adenoma cell adhesion and migration.

\section{Discussion}

ADAM10 is a membrane-anchored member of the ADAM family and is ubiquitously expressed throughout most tissues, including neurons, the vasculature, leukocytes, and tumor cells (Moss et al. 2008). ADAM10 has been reported to be crucial for developmental processes, based on the data from mice with impaired Adam10 expression (Hartmann et al. 2002). However, the most important function of ADAM10 is still considered to be its proteinase activity. A consensus sequence of HEXGHXXGXXHD is located within the active sites in the metalloproteinase domain of the proteinase-type ADAM molecules. This sequence is also present in the catalytic metalloproteinase domain of 
A

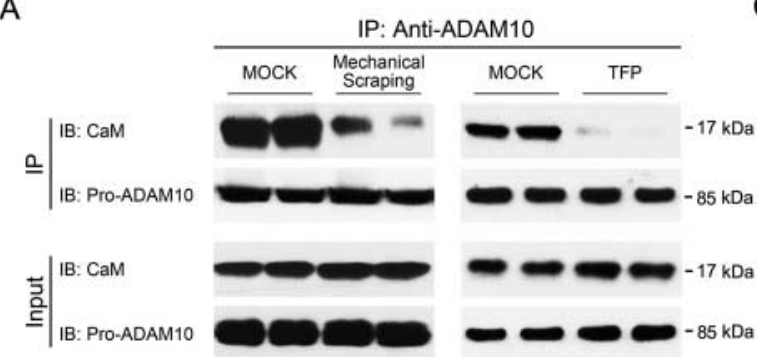

B
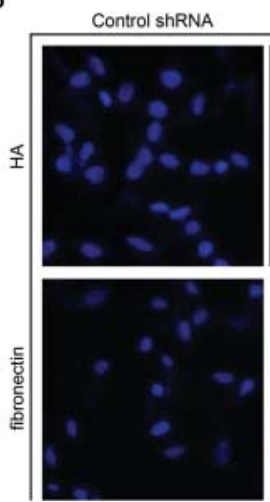

ShADAM10

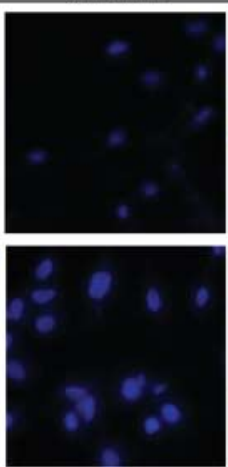

ShADAM10+CD44EXT

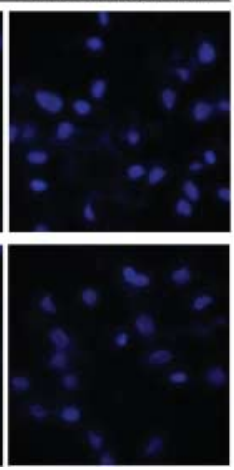

C

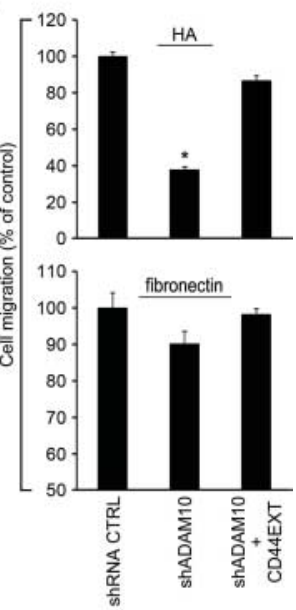

Figure 5 ADAM10 mediates AtT-20 cell migration in a CD44-dependent manner. (A) The interaction of CaM with Pro-ADAM10 decreases after mechanical scraping or TFP treatment. (B) CD44EXT overexpression reversed the effects of Adam10 knockdown on migration of AtT-20 cells under the hyaluronic acid-coated condition. (C) Quantitative measurement of the migration of AtT-20 cells. Bars show mean \pm s.D. ${ }^{*} P<0 \cdot 01$.

All experiments were repeated three times.

MMP family members (Mochizuki \& Okada 2007). Through its proteinase activity, ADAM10 can process several critical signaling molecules associated with cancer development and tumor progression, including Her2, CD44, and L1 (Moss et al. 2008). These molecules play important roles in cell adhesion and migration, which are widely known to be the key steps in tumor invasion. Overexpression of ADAM10 has been found in oral cavity (Ko et al. 2007), stomach (Yoshimura et al. 2002), ovary, uterine (Fogel et al. 2003), colon (Gavert et al. 2005), leukemia (Wu et al. 1997), and prostate (McCulloch et al. 2004) carcinoma patients. ADAM10 has been reported to participate in the proliferation of some tumor cells; overexpression of ADAM10 can promote cell proliferation, and blocking ADAM10 expression reduces cell growth (Carl-McGrath et al. 2005, Ko et al. 2007). Some studies have indicated that the stimulation of proliferation mediated by ADAMs is involved in early tumorigenesis, possibly by releasing growth factors or allowing the cells to escape immune surveillance (Sanderson et al. 2006, Moss et al. 2008). However, more details regarding the functions of ADAM10 in cancers remain to be elucidated.

In this study, we first identified that ADAM1O expression is higher in high-grade pituitary adenoma patients and is associated with the cleavage of L1 and CD44. In addition, we found that two important signaling molecules, CaM and Src, present abnormal interactions with ADAM10 and Shc respectively. In high-grade pituitary adenomas or a stimulated pituitary adenoma cell line, further investigation indicated that L1 and CD44 are shed by ADAM10 through different signals; CaM releases its suppression of ADAM10 to promote CD44 cleavage, whereas increased Src/ERK1/2 signal leads to increased L1 shedding. These results demonstrate that adhesion molecules are involved in promoting tumor invasiveness through ADAM10.

L1 can be cleaved constitutively at the cell surface, and this process is enhanced by stimulation of PMA. Overexpression of L1 has been found in many types of tumors and is associated with enhanced invasiveness and metastasis (Ohnishi et al. 1998, Mechtersheimer et al. 2001, Thies et al. 2002). Interestingly, ADAM10 is overexpressed at the invasive edge of many cancers, where L1 overexpression is also observed (Fogel et al. 2003, Gavert et al. 2005). Soluble L1 has been shown to enhance cell migration in vitro, which might be mediated by binding to integrins (Mechtersheimer et al. 2001). Metalloprotease inhibitors can block cell 
migration mediated by L1. In neuronal cells, L1-dependent adhesion and haptotactic migration are blocked when L1 shedding is inhibited (Maretzky et al. 2005). Some ADAMs contain SH3-binding sites in their cytoplasmic tail. Therefore, a series of SH3domain-containing signaling factors might be activated by ADAMs, such as Src and its downstream MAPK pathway (Barberis et al. 2000, Seals \& Courtneidge 2003, Kleiner et al. 2007). Our data show that the activation of Src and ERK1/2 correlates with ADAM10 expression in high-grade pituitary adenoma patients. ADAM10mediated L1 cleavage is blocked by the inhibitors of Src and MEK. Therefore, one might speculate that these signaling molecules are activated in high-grade pituitary adenoma patients and modify the intracytoplasmic tail domains of ADAMs, which leads to conformational changes and enables them to interact with specific substrates (Mochizuki \& Okada 2007). However, these possibilities remain to be elucidated by further studies.

CD44 also mediates tumor cell migration and invasion, and its function might be regulated by ADAM10 on several different levels (Nagano et al. 2004). ADAM10 contributes to the production of soluble CD44 by ectodomain cleavage. This process is enhanced by calcium ionophores. Soluble CD44 has been reported in various tumor cells, and in many cases this phenomenon correlates with decreased cell adhesion and increased detachment of the cells from the ECM (Nagano et al. 2004, Moss et al. 2008). An explanation for this correlation is that the shedding of CD44 reduces the density of the surface molecule and soluble CD44 acts as an antagonist of transmembrane CD44 by binding to extracellular hyaluronan and reducing cell adhesion (Okamoto et al. 2002, Murakami et al. 2003). Another consequence of ADAM10-dependent CD44 shedding is the release of a cytosolic fragment, which regulates gene expression, including the expression of CD44 itself (Nagano $\mathrm{et} \mathrm{al}$. 2004). Thus, this type of feedback for CD44 expression may further amplify CD44 activity in cancer cells.

Calcium is considered to contribute to increased cell mobility through blocking cell-matrix adhesion. Recently, many studies have indicated that extracellular $\mathrm{Ca}^{2+}$ influx promotes the shedding of many proteins, including CD44 and E-cadherin (Ito et al. 1999). $\mathrm{Ca}^{2+}$-influx-induced ectodomain cleavage is mediated by ADAM10 metalloproteinases. CaM, which suppresses ADAM10 activity through protein interaction (Nagano et al. 2004), dissociates from ADAM10 under calcium overload and subsequently enhances CD44 shedding and CD44-dependent cell migration.

Our study demonstrates the molecular mechanism of how ADAM10 participates in the invasiveness of pituitary adenomas through the regulation of adhesion factors. However, distinct mechanisms are involved in this process. The changes in some proinflammatory factors, such as tumor necrosis factor- $\alpha$, epidermal growth factor, Fas ligand, cytokines, and chemokines, require further investigation. While features, such as necrosis, mitosis, and hemorrhage, are unreliable markers of the invasiveness of these tumors, ADAM10 may be a useful molecule for determining invasiveness. Notably, an ADAM10 inhibitor could potentially represent a beneficial method to treat cancers. Recently, more attention has been focused on ADAM10 inhibitors because of their ability to inhibit ErbB signaling (Moss et al. 2008). Therefore, the development of specific ADAM10 inhibitors that target pathogenic shedding events will be a promising strategy in cancer therapy.

MMPs are important in digestion of the ECM during tumor invasion and metastasis. Many researches have illustrated that MMP2 (Liu et al. 2005) and MMP9 (Gong et al. 2008) play important roles in pituitary tumor invasion. Both MMP2 and MMP9 are type IV collagenases, which can break down basement membrane, in particular, cleaving type IV collagen (Kawamoto et al. 1996). Like MMP2 and MMP9, ADAM10 is also a type IV collagenase (Millichip et al. 1998). As we mentioned earlier, ADAM10 expression increased in various types of cancer (Wu et al. 1997, Yoshimura et al. 2002, Fogel et al. 2003, McCulloch et al. 2004, Gavert et al. 2005, Ko et al. 2007). Here, we found that $A D A M 10$ expression is enhanced in pituitary adenomas and associated with the invasiveness of pituitary adenomas, and further investigation revealed that ADAM10 facilitated cell migration through modulation of $\mathrm{CD} 44$ and $\mathrm{L} 1$ cleavage. These results discovered a new mechanism of pituitary adenoma invasiveness and indicated new potential therapeutic targets for pituitary adenomas.

\section{Declaration of interest}

The authors declare that there is no conflict of interest that could be perceived as prejudicing the impartiality of the research reported.

\section{Funding}

This work was supported by the Shanghai Municipal Science and Technology Commission (No. 10JC1418200) and the National Natural Science Foundation of China (No. 30930094).

\section{References}

Allinson TM, Parkin ET, Turner AJ \& Hooper NM 2003 ADAMs family members as amyloid precursor protein $\alpha$-secretases. Journal of Neuroscience Research 74 342-352. (doi:10.1002/jnr.10737)

Arribas J, Bech-Serra JJ \& Santiago-Josefat B 2006 ADAMs, cell migration and cancer. Cancer and Metastasis Reviews 25 57-68. (doi:10.1007/s10555-006-7889-6) 
Barberis L, Wary KK, Fiucci G, Liu F, Hirsch E, Brancaccio M, Altruda F, Tarone G \& Giancotti FG 2000 Distinct roles of the adaptor protein Shc and focal adhesion kinase in integrin signaling to ERK. Journal of Biological Chemistry 275 36532-36540. (doi:10.1074/jbc. M002487200)

Blobel CP 1997 Metalloprotease-disintegrins: links to cell adhesion and cleavage of TNF $\alpha$ and Notch. Cell 90 589-592. (doi:10.1016/ S0092-8674(00) 80519-X)

Borrell-Pages M, Rojo F, Albanell J, Baselga J \& Arribas J 2003 TACE is required for the activation of the EGFR by TGF- $\alpha$ in tumors. EMBO Journal 22 1114-1124. (doi:10.1093/emboj/cdg111)

Brummendorf T, Kenwrick S \& Rathjen FG 1998 Neural cell recognition molecule L1: from cell biology to human hereditary brain malformations. Current Opinion in Neurobiology 8 87-97. (doi:10.1016/S0959-4388(98)80012-3)

Bulayeva NN \& Watson CS 2004 Xenoestrogen-induced ERK-1 and ERK-2 activation via multiple membrane-initiated signaling pathways. Environmental Health Perspectives 112 1481-1487. (doi:10.1289/ehp.7175)

Carl-McGrath S, Lendeckel U, Ebert M, Roessner A \& Rocken C 2005 The disintegrin-metalloproteinases ADAM9, ADAM12, and ADAM15 are upregulated in gastric cancer. International Journal of Oncology 26 17-24.

Crawford HC, Dempsey PJ, Brown G, Adam L \& Moss ML 2009 ADAM10 as a therapeutic target for cancer and inflammation. Current Pharmaceutical Design 15 2288-2299. (doi:10.2174/ 138161209788682442)

Daniel L, Trouillas J, Renaud W, Chevallier P, Gouvernet J, Rougon G \& Figarella-Branger D 2000 Polysialylated-neural cell adhesion molecule expression in rat pituitary transplantable tumors (spontaneous mammotropic transplantable tumor in Wistar-Furth rats) is related to growth rate and malignancy. Cancer Research 60 80-85.

Egeblad M \& Werb Z 2002 New functions for the matrix metalloproteinases in cancer progression. Nature Reviews. Cancer 2 161-174. (doi:10.1038/nrc745)

Fogel M, Gutwein P, Mechtersheimer S, Riedle S, Stoeck A, Smirnov A, Edler L, Ben-Arie A, Huszar M \& Altevogt P 2003 L1 expression as a predictor of progression and survival in patients with uterine and ovarian carcinomas. Lancet 362 869-875. (doi:10.1016/S01406736(03) 14342-5)

Gavert N, Conacci-Sorrell M, Gast D, Schneider A, Altevogt P, Brabletz T \& Ben-Ze'ev A 2005 L1, a novel target of $\beta$-catenin signaling, transforms cells and is expressed at the invasive front of colon cancers. Journal of Cell Biology 168 633-642. (doi:10.1083/ jcb.200408051)

Gong J, Zhao Y, Abdel-Fattah R, Amos S, Xiao A, Lopes MB, Hussaini IM \& Laws ER 2008 Matrix metalloproteinase-9, a potential biological marker in invasive pituitary adenomas. Pituitary 11 37-48. (doi:10.1007/s11102-007-0066-2)

Gunthert AR, Strater J, von Reyher U, Henne C, Joos S, Koretz K, Moldenhauer G, Krammer PH \& Moller P 1996 Early detachment of colon carcinoma cells during CD95(APO-1/Fas)-mediated apoptosis. I. De-adhesion from hyaluronate by shedding of CD44. Journal of Cell Biology 134 1089-1096. (doi:10.1083/jcb.134.4.1089)

Gutwein P, Oleszewski M, Mechtersheimer S, Agmon-Levin N, Krauss K \& Altevogt P 2000 Role of Src kinases in the ADAM-mediated release of $\mathrm{L} 1$ adhesion molecule from human tumor cells. Journal of Biological Chemistry 275 15490-15497. (doi:10.1074/jbc.275.20. 15490)

Hartmann D, de Strooper B, Serneels L, Craessaerts K, Herreman A, Annaert W, Umans L, Lubke T, Lena Illert A, von Figura K et al. 2002 The disintegrin/metalloprotease ADAM 10 is essential for Notch signalling but not for $\alpha$-secretase activity in fibroblasts. Human Molecular Genetics 11 2615-2624. (doi:10.1093/hmg/11.21.2615)

Hortsch M 2000 Structural and functional evolution of the L1 family: are four adhesion molecules better than one? Molecular and Cellular Neuroscience 15 1-10. (doi:10.1006/mcne.1999.0809)
Ito K, Okamoto I, Araki N, Kawano Y, Nakao M, Fujiyama S, Tomita K, Mimori T \& Saya H 1999 Calcium influx triggers the sequential proteolysis of extracellular and cytoplasmic domains of E-cadherin, leading to loss of $\beta$-catenin from cell-cell contacts. Oncogene $\mathbf{1 8}$ 7080-7090. (doi:10.1038/sj.onc.1203191)

Kawamoto H, Uozumi T, Arita K, Yano T \& Hirohata T 1996 Type IV collagenase activity and cavernous sinus invasion in human pituitary adenomas. Acta Neurochirurgica 138 390-395. (doi:10.1007/ BF01420300)

Kenny PA \& Bissell MJ 2007 Targeting TACE-dependent EGFR ligand shedding in breast cancer. Journal of Clinical Investigation 117 337-345. (doi:10.1172/JCI29518)

Kirkegaard T, Edwards J, Tovey S, McGlynn LM, Krishna SN, Mukherjee R, Tam L, Munro AF, Dunne B \& Bartlett JM 2006 Observer variation in immunohistochemical analysis of protein expression, time for a change? Histopathology 48 787-794. (doi:10.1111/j.1365-2559.2006.02412.x)

Kleiner S, Faisal A \& Nagamine Y 2007 Induction of uPA gene expression by the blockage of E-cadherin via Src- and Shc-dependent Erk signaling. FEBS Journal 274 227-240. (doi:10.1111/j.1742-4658.2006.05578.x)

Knosp E, Steiner E, Kitz K \& Matula C 1993 Pituitary adenomas with invasion of the cavernous sinus space: a magnetic resonance imaging classification compared with surgical findings. Neurosurgery 33 610-617 (discussion 617-618). (doi:10.1227/00006123199310000-00008)

Ko SY, Lin SC, Wong YK, Liu CJ, Chang KW \& Liu TY 2007 Increase of disintergin metalloprotease 10 (ADAM10) expression in oral squamous cell carcinoma. Cancer Letters 245 33-43. (doi:10.1016/j. canlet.2005.10.019)

Lammich S, Kojro E, Postina R, Gilbert S, Pfeiffer R, Jasionowski M, Haass C \& Fahrenholz F 1999 Constitutive and regulated $\alpha$-secretase cleavage of Alzheimer's amyloid precursor protein by a disintegrin metalloprotease. PNAS 96 3922-3927. (doi:10.1073/pnas.96.7.3922)

Liu WP, Kunishio K, Matsumoto Y, Okada M \& Nagao S 2005 Matrix metalloproteinase-2 expression correlates with cavernous sinus invasion in pituitary adenomas. Journal of Clinical Neuroscience 12 791-794. (doi:10.1016/j.jocn.2005.03.010)

Maretzky T, Schulte M, Ludwig A, Rose-John S, Blobel C, Hartmann D, Altevogt P, Saftig P \& Reiss K 2005 L1 is sequentially processed by two differently activated metalloproteases and presenilin/gammasecretase and regulates neural cell adhesion, cell migration, and neurite outgrowth. Molecular and Cellular Biology 25 9040-9053. (doi:10.1128/MCB.25.20.9040-9053.2005)

McCulloch DR, Akl P, Samaratunga H, Herington AC \& Odorico DM 2004 Expression of the disintegrin metalloprotease, ADAM-10, in prostate cancer and its regulation by dihydrotestosterone, insulin-like growth factor I, and epidermal growth factor in the prostate cancer cell model LNCaP. Clinical Cancer Research 10 314-323. (doi:10.1158/1078-0432.CCR-0846-3)

Mechtersheimer S, Gutwein P, Agmon-Levin N, Stoeck A, Oleszewski M, Riedle S, Postina R, Fahrenholz F, Fogel M, Lemmon V et al. 2001 Ectodomain shedding of L1 adhesion molecule promotes cell migration by autocrine binding to integrins. Journal of Cell Biology 155 661-673. (doi:10.1083/jcb.200101099)

Millichip MI, Dallas DJ, Wu E, Dale S \& McKie N 1998 The metallo-disintegrin ADAM10 (MADM) from bovine kidney has type IV collagenase activity in vitro. Biochemical and Biophysical Research Communications 245 594-598. (doi:10.1006/bbrc.1998. 8485)

Mochizuki S \& Okada Y 2007 ADAMs in cancer cell proliferation and progression. Cancer Science 98 621-628. (doi:10.1111/j.1349-7006. 2007.00434.x)

Moss ML, Stoeck A, Yan W \& Dempsey PJ 2008 ADAM10 as a target for anti-cancer therapy. Current Pharmaceutical Biotechnology 9 2-8. (doi:10.2174/138920108783497613)

Murai T, Miyazaki Y, Nishinakamura H, Sugahara KN, Miyauchi T, Sako Y, Yanagida T \& Miyasaka M 2004 Engagement of CD44 promotes 
Rac activation and CD44 cleavage during tumor cell migration. Journal of Biological Chemistry 279 4541-4550. (doi:10.1074/jbc. M307356200)

Murakami D, Okamoto I, Nagano O, Kawano Y, Tomita T, Iwatsubo T, De Strooper B, Yumoto E \& Saya H 2003 Presenilin-dependent gamma-secretase activity mediates the intramembranous cleavage of CD44. Oncogene 22 1511-1516. (doi:10.1038/sj.onc.1206298)

Nagano O, Murakami D, Hartmann D, De Strooper B, Saftig P, Iwatsubo T, Nakajima M, Shinohara M \& Saya H 2004 Cell-matrix interaction via CD44 is independently regulated by different metalloproteinases activated in response to extracellular $\mathrm{Ca}(2+)$ influx and PKC activation. Journal of Cell Biology 165 893-902. (doi:10.1083/jcb.200310024)

Ohnishi T, Matsumura H, Izumoto S, Hiraga S \& Hayakawa T 1998 A novel model of glioma cell invasion using organotypic brain slice culture. Cancer Research 58 2935-2940.

Okamoto I, Tsuiki H, Kenyon LC, Godwin AK, Emlet DR, Holgado-Madruga M, Lanham IS, Joynes CJ, Vo KT, Guha A et al. 2002 Proteolytic cleavage of the CD44 adhesion molecule in multiple human tumors. American Journal of Pathology 160 441-447. (doi:10.1016/S0002-9440(10)64863-8)

Parkin E \& Harris B 2009 A disintegrin and metalloproteinase (ADAM)-mediated ectodomain shedding of ADAM10. Journal of Neurochemistry 108 1464-1479. (doi:10.1111/j.1471-4159.2009. 05907.x)

Pruessmeyer J \& Ludwig A 2009 The good, the bad and the ugly substrates for ADAM10 and ADAM17 in brain pathology, inflammation and cancer. Seminars in Cell E Developmental Biology 20 164-174. (doi:10.1016/j.semcdb.2008.09.005)

Qian ZR, Sano T, Yoshimoto K, Asa SL, Yamada S, Mizusawa N \& Kudo E 2007 Tumor-specific downregulation and methylation of the CDH13 (H-cadherin) and CDH1 (E-cadherin) genes correlate with aggressiveness of human pituitary adenomas. Modern Pathology 20 1269-1277. (doi:10.1038/modpathol. 3800965)

Sanderson MP, Dempsey PJ \& Dunbar AJ 2006 Control of ErbB signaling through metalloprotease mediated ectodomain shedding of EGF-like factors. Growth Factors 24 121-136. (doi:10.1080/ 08977190600634373)
Santiago-Josefat B, Esselens C, Bech-Serra JJ \& Arribas J 2007 Posttranscriptional up-regulation of ADAM17 upon epidermal growth factor receptor activation and in breast tumors. Journal of Biological Chemistry 282 8325-8331. (doi:10.1074/jbc.M608826200)

Schachner M 1997 Neural recognition molecules and synaptic plasticity. Current Opinion in Cell Biology 9 627-634. (doi:10.1016/ S0955-0674(97)80115-9)

Seals DF \& Courtneidge SA 2003 The ADAMs family of metalloproteases: multidomain proteins with multiple functions. Genes and Development 17 7-30. (doi:10.1101/gad.1039703)

Thies A, Schachner M, Moll I, Berger J, Schulze HJ, Brunner G \& Schumacher U 2002 Overexpression of the cell adhesion molecule $\mathrm{L} 1$ is associated with metastasis in cutaneous malignant melanoma. European Journal of Cancer 38 1708-1716. (doi:10.1016/S09598049(02)00105-3)

White JM 2003 ADAMs: modulators of cell-cell and cell-matrix interactions. Current Opinion in Cell Biology 15 598-606. (doi:10.1016/j.ceb.2003.08.001)

Wu E, Croucher PI \& McKie N 1997 Expression of members of the novel membrane linked metalloproteinase family ADAM in cells derived from a range of haematological malignancies. Biochemical and Biophysical Research Communications 235 437-442. (doi:10.1006/ bbrc.1997.6714)

Yoshimura T, Tomita T, Dixon MF, Axon AT, Robinson PA \& Crabtree JE 2002 ADAMs (a disintegrin and metalloproteinase) messenger RNA expression in Helicobacter pylori-infected, normal, and neoplastic gastric mucosa. Journal of Infectious Diseases $\mathbf{1 8 5}$ 332-340. (doi:10.1086/338191)

Zhou BB, Peyton M, He B, Liu C, Girard L, Caudler E, Lo Y, Baribaud F, Mikami I, Reguart N et al. 2006 Targeting ADAM-mediated ligand cleavage to inhibit HER3 and EGFR pathways in nonsmall cell lung cancer. Cancer Cell 10 39-50. (doi:10.1016/j.ccr.2006.05.024)

Received in final form 11 May 2012

Accepted 14 May 2012

Made available online as an Accepted Preprint 14 May 2012 